JAIST Repository

https://dspace.jaist.ac.jp/

\begin{tabular}{|c|c|}
\hline Title & $\begin{array}{l}\text { Desi gn of a Sof tware Agent for Busi ness Gaming } \\
\text { Si mul at i on }\end{array}$ \\
\hline Author(s) & $\begin{array}{l}\text { Shi noda, Yuj i ; Ryoke, M na; Ter ano, Takao; } \\
\text { Nakanmr i , Yoshi ter u }\end{array}$ \\
\hline \multicolumn{2}{|l|}{ Citation } \\
\hline Issue Date & $2005-11$ \\
\hline Type & Conf er ence Paper \\
\hline Text version & publ i sher \\
\hline URL & ht t p: //hdl . handl e. net /10119/3868 \\
\hline Rights & (C)2005 JAl ST Press \\
\hline Description & 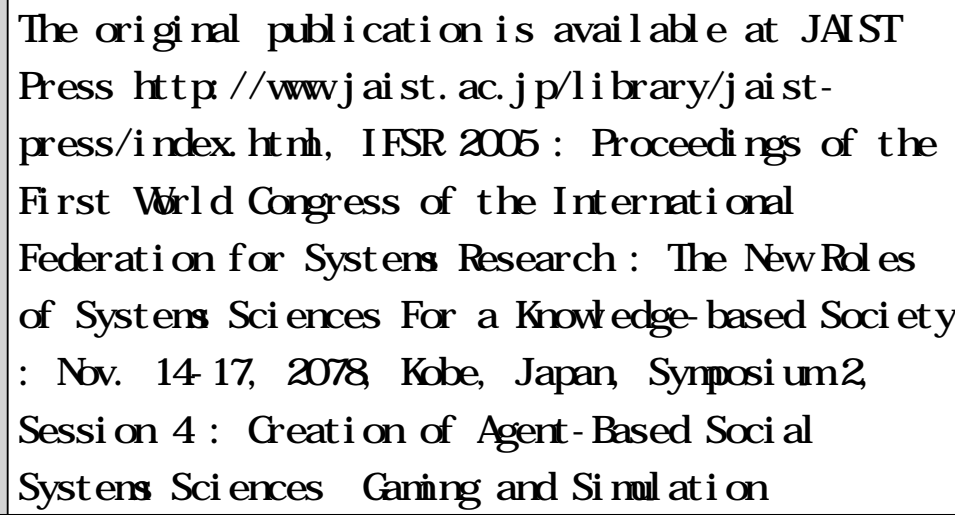 \\
\hline
\end{tabular}

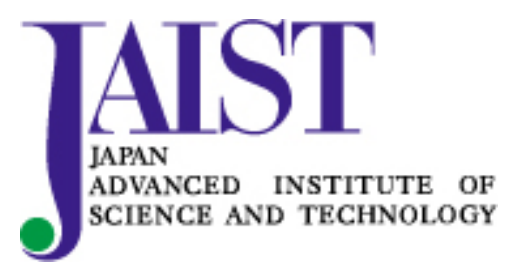




\title{
Design of a Software Agent for Business Gaming Simulation
}

\author{
Y. Shinoda ${ }^{1}$, M. Ryoke ${ }^{2}$, T. Terano ${ }^{3}$, and Y. Nakamori ${ }^{1}$ \\ ${ }^{1}$ School of Knowledge Science, Japan Advanced Institute of Science and Technology \\ 1-1 Asahidai, Nomi, Ishikawa 923-1292, Japan \\ y-shino@jaist.ac.jp \\ ${ }^{2}$ Graduate School of Business Sciences, University of Tsukuba, Tokyo \\ 3-29-1 Otsuka, Bunkyo, Tokyo 112-0012, Japan \\ ${ }^{3}$ Dept. Computational Intelligence and Systems Science, Tokyo Institute of Technology \\ 4259 Nagatsuda-cho, Midori-ku, Yokohama 226-8503, Japan
}

\begin{abstract}
This paper introduces an agent model for one of the business games called Bakery Game. Business game is a tool the understand business and the principle of management through the experience in a virtual world. In order to construct computer agents for the gaming simulation that can participate in the game and become human players' worthy rival, we combine simple model that consider the property of Bakery Game and strategy adjustment model. The agent has so-called strategy parameters that are updated through the experience. The agent changes the strategy depending on the situation. The agent participates in the game that regenerated from log data that recorded various situations.
\end{abstract}

Keywords: gaming simulation, strategy searching, agent based simulation

\section{INTRODUCTION}

\subsection{Gaming Simulation and Business Game}

When we have to decide something, we usually must think about other people. The result of our decision-making might be changed other people's decisions. To think of and study this kind of situation, we face to gaming simulation. Stahl showed that there are five types of gaming simulation. Entertainment games, Educational games, Experimental games, Research games, and Operational games [1]. In the field of Educational games, there is Business game.

Business game is a method that focuses on understanding about business and the principle of the management through the experience in a virtual world [2]. Because of the following advantage, Business Gaming is used in business and education environments. From the point of view of Gaming Player, player can have experience in a virtual world. Player can test his ideas and strategies in the game in order to understand deeply and to find some new ideas [3]. From the point of view of Gaming Designer, the designer has three kinds of merit. The first one is that the game designer can observe his gaming model carefully and construct his model again through business gaming. The second one is the gaming designer may find out new player's strategy through observing business gaming. The third one is that game designer can check whether his game can express his opinion to players or not [4]. As one of the demerits on business gaming, game players are required to play game with efforts. Most of business games have some complex items that should be decided by the players. Some games require players to prepare for the game and to play for long time. This sometimes leads to a lack of players. If the game will become too simple without having enough players, the lack of players causes problem. Because of these problems, the game facilitator wants pinch hitter of the player.

The Graduate School of Systems Management in Tsukuba University uses a business gaming system as education tool for students [2] [5]. The gaming system is constructed on the WWW and thus avoiding the problem on the distance between players. The maximum number of the player is 12 teams in the standard. However, preparation of enough players is not easy. In such a case, the absent teams are removed from the game or use regular parameters decided by the game designer. The regular parameters are fixed and that enables human players easily to find that there are the non-thinking teams. These non-thinking teams just consume certain pie of the market without tactics. To avoid these problems, computer agents [6] may be used as players for gaming simulation.

\subsection{Agents}

Kobayashi et al [7] shows a gaming agent for GSMM gaming system using a classification system. The agent has a learning function to find good strategy in a 
business game. Baba et al [8] show that neural-network system can learn a player's behavior, and the neural-network system can play a game same as the original player. They also show that a genetic algorithm based program can defeat a human player in one-by-one game. In these previous work, agents can become strong players, but they are not focused on to adopt a game in real-time. In [9], we proposed an agent that based on game designer's strategy in real-time. However, this agent model uses the game model that is hidden from players in order to evaluate agent's strategy and the agent cannot win in a game with human players.

The difficulty of the agent model for a business game is as follows. The same game, the same member, and same demands of the market, the result of the game may be different. This is because the decision-making of players influences the market. This makes it difficult to construct an agent player. Moreover, in the field of education, the number of the players and the tendency of the demands are not always same. There are also differences on the understanding of the game model between players. These problems also make it difficult to construct the agent model.

In this paper, we propose an agent algorithm of gaming simulation that adjusts its strategy in real-time by using the experience of the past gaming.

\subsection{Contents of this paper}

The content of this paper is as follows. Section 2 introduces the Bakery Game, which is our main target. Section 3 describes the agent's decision-making model, and introduces the agent's strategy model though the log data. In Section 4, we set up the simulator and test the algorithm. Our conclusions and issues for future work are described in Section 5.

\section{BAKERY GAME}

\subsection{Bakery Game}

This paper focuses on one of the business game that is called Bakery Game. Bakery Game is developed in Yokohama University. In Bakery Game, player becomes a manager of Bakery, and players compete about their surplus money through a game. Players buy frozen dough from dough factory. Then players defrost and bake the dough. Players sell the baked bread, and unsold bread will be thrown away after the round. Game master defines the total amount of customers. Depending upon the price of the bread, the customers are distributed to the bakeries. Players must care about time lag during buying of frozen dough and defrosting the dough. Through Bakery Game, players can learn about decision-making, merchandise and demand prediction under a time lag.

The game is divided into rounds. In each round, the player makes their decision. Players are called as team 1 , team 2, and team 3.... In the beginning of each round, the player makes three kind of decision, stock amount, defrost amount, and price. The procedure of each round is in the following.

1. The certain amount of bread that was decided by the player in the previous round is completely defrosted and baked.

2. The customers are distributed depending on the prices that are decided by the players in this round. The amount of orders from customer to each player is calculated.

3. The certain amount of dough that was ordered in previous round arrives to the players' warehouse and the player pays the cost of the dough.

4. The certain amount of dough that is decided as defrost amount in this round begins defrosting.

5. The certain amount of dough that was decided as the order amount in this round is ordered to dough factory.

Figure 1 shows the outline of Bakery Game. The amount of order from customer is not directly meaning that whole customer can buy bread. The amount of the bread in the bakery is limited. If the amount of order surpasses the amount of the bread in the bakery, the remainder order is lost. Depending on the amount of loss, the customer of the player is decreased as the penalty in the next round. After the round, players can know all of the prices of the teams and how many customers went to each bakery.
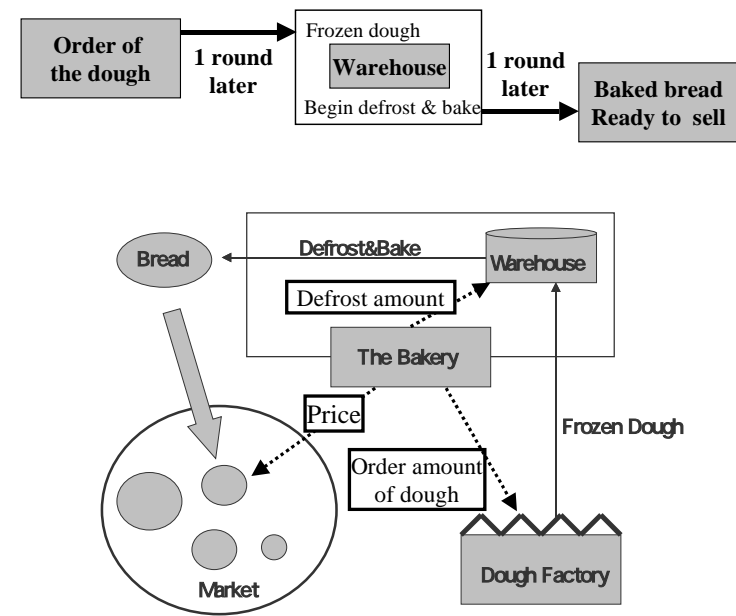

Fig. 1 Outline of Bakery Game 


\begin{abstract}
Game Scenario-
You become the manager of a bakery in your town by renting your bakery. There are 10 bakeries in this town, and the quality of the bread is the same. Demand for the bread is 1300 loaves/day, and it seems to be increasing. Usually, the bread is sold at 700 yen/loaf. The customers like cheaper bread, so decreasing the price of the bread will increase the number of customers.
\end{abstract}

To sell the bread, you must defrost frozen dough and bake it, and this process takes a day. You can buy the frozen dough from a dough factory. After your order, the dough is delivered to your bakery the next day. If you do not bake enough bread, you lost sales and you might also lose customers because of the bad service of your bakery. On the other hand, if you bake too much bread, the unsold bread must be thrown away. You can keep your frozen dough in the warehouse.

You can buy a loaf of frozen dough for 400 yen. You must pay the cost of dough when it delivered. You bake one loaf of bread from a loaf of dough, and the cost of baking is 100 yen/loaf, which includes the salary of a person to bake the dough. To rent your bakery, you need 20000 yen per day. The income of your bakery is cash from the bread that is sold.

The above scenario is given to players. There is a model that distributes customers to the bakeries. However, the distribution model is hidden from players to simulate a bakery in the real world.

\subsection{Gaming Model}

The Bakeries are called team 1, team 2, and team 3 . The distribution of the customers is written as follows.

$C_{t}(i)=\frac{\left(P_{t}(i)-P m\right)^{3}}{\sum\left(\left(P_{t}(i)-P m\right)^{3}\right)} C_{t}$

Here, $P_{t}(i)$ is the price set by team $i$ in time $t$, $C_{t}(i)$ is the number of the distributed customers of team $i$ in time $t . C_{t}$ is the total number of customers in the market in time $t . P m$ is the lower limit of the price that was decided by the gaming system.

The next equation shows a penalty in which the number of customers decreases because of bad service when the bakery's bread sold out. The sold out penalty $\mathrm{Cmp}_{t}(\mathrm{i})$ of team $i$ in time $t$ is calculated by the following equation.

$$
\begin{aligned}
& \operatorname{Cmp}_{t}(i)=\left(C_{t-1}(i)-D_{t-1}\right) \times 0.5 \\
& \text { if } \\
& \left\{\left(C_{t-1}(i)-D_{t-1}\right) \geq 0\right\}
\end{aligned}
$$

Here , $D_{t}$ is the amount defrosted by team $i$ in time $t$. If the agent has been penalized for having more customers than bread, then the excess number of customers will be subtracted from the distributed customers in the next round.

\subsection{View Point of the Game Designer}

The base of the bakery game is the ideas that a player at least must try to get same share as other players, and a player at least must try to make a profit at least the upkeep cost of the shop. Furthermore, to win the game, a player must decrease possibility of missing chance. For example, the player must decrease the waste of the bread because of the unsold, and decrease the lost of customer because of the sellout.

The designer's procedure is the following. The player must predict the size of the market under the assumption that customers are distributed to all bakeries, the player can predict the average number of customer in the current round. Then the player must decide supposed amount of customers thinking his strategy into account. With the supposed amount of customers, the player decides the defrost amount of the dough. Next, the player decides the price in this round. Now in his bakery, there are baked bread that he decided as the defrost amount in the previous round. The player must decide the optimal price of the bread to avoid the sellout and the unsold. Considering the upkeep cost of his bakery, the player needs to keep the minimum price that divided the upkeep cost with the amount of the bread. The player can add certain profit margin to the base price to decide the price of this round. This profit margin includes his strategy. Finally, the amount of stock should be decided under consideration of the change of the defrost amount and the price and the players strategy.

Above procedure is the method that the designer recommends. From this method, the game designer divides the game model to three sections; price, defrost, stock. These concepts are connected by strategic viewpoint. In the next section we describe our decision-making model. We use this three-divided structure in our agent model in the next section. 


\section{DECISION-MAKING MODEL}

\subsection{The Agent's Strategy}

The word 'strategy' is widely used. One of its meanings in the management field is the framework for deciding the company's objectives. In this framework, it is necessary to handle two problems: what problems the decision-maker solves and how they are solved [10]. Others would include information gathering and analysis as a third problem to be included in the framework [11]. In the world of game that is constructed in the business game, the strategy is also constructed from the forecast of the market and its analyses, the setting of the problem, and the tactics available to solve the problem.

In this research, we propose an agent model that has a decision-making model and a strategy adjustment model. By using basic decision-making model, the agent calculates clues of the game. These clues are just based on the game status in the previous rounds, and there is no prediction of the market or plans for future benefit. Agent adjusts the clues from strategic viewpoint and makes decision with strategy adjustment model. Therefore, the word "strategy" in this research does not have meaning as a framework. We deal strategy as a set of situation and answers to operate the specified decision-making model.

\subsection{The Agent's Decision-making Model}

The bakery game is a game that competes for the surplus. The gain of the surplus can be calculated according to the price of dough, the baking cost, and the upkeep cost of the bakery. There is a certain combination of the number of the baked bread and the price that achieves maximum gain when the bread can be prepared without the excess and deficiency. We try to construct agent's decision-making model with the property of this game model based on the game designer decision-making model. However, the situation of the game changes depending on the decision-making of other players and the change of the total demand of the game. To win a game, it is not enough to know the best combination of the number of the bread and price. Human players can consider from a strategic viewpoint including his experiences about the risk. The agent should study fine-tuning this strategy through the games. We now propose a new decision-making model as follows.

From the public information in the previous round of the game, the agent generates a model which describes the relation between price and the number of customers (model A), and which a model which describes the relation between the number of customer and the gain (model B). Using model A, the agent can find the other price that maximizes the gain. The Agent's decision-making is written as follows.

$P_{t}=F p c\left(B_{t}\right) \times \alpha$

Here, $P_{t}$ is the price and $B_{t}$ is the amount of the bread in the shop in round $t, F p c(X)$ is the optimal price calculated by model A with $X$ customers, and $\alpha$ is the strategic parameter of price.

The defrost amount is based on the Number of customers-Gain model with considering the total demand in the previous two rounds.

$$
\begin{aligned}
& D_{t}=C g_{t} \times C r_{t} \times \beta \\
& C r_{t}=\frac{C_{t-1}}{C_{t-2}}
\end{aligned}
$$

Here, $D_{t}$ is the defrost amount in round $t . C g_{t}$ is the optimal number of the customer calculated by model B. $\beta$ is the strategic parameter of defrost. $C_{t}$ is the number of total customer in the market and $C r_{t}$ is the customer changing ratio in round $t$.

Stocking the frozen dough indirectly influences the price. We introduce the following rule that allows the agent keeping a certain amount of dough in the warehouse above the defrost amount.

The defrosting strategy is written as the following equation.

$$
S t_{t}+W_{t}=\gamma \times D_{t}
$$

Here, $S t_{t}$ is the stock amount in time $t . W_{t}$ means the frozen dough in the warehouse at the beginning of the round. $\gamma$ is the strategic parameter of stock. Formula (6) is the stock strategy in which the agent keeps a certain amount of dough in the warehouse against the defrost amount. If $\gamma$ is equal to 1.2 , the agent stocks 1.2 times the amount of defrosting dough in this round in the warehouse at the next round. The agent prepares the commodity corresponding to an increase in demand. 
Particularly we now set numerical values for the strategic parameters that are included in the decision-making rule of the agent. $\boldsymbol{\alpha}$, the strategic parameter for price, is selected from following 7 values: $97.5 \%, 100 \%, 102.5 \%, 105 \%, 107.5 \%, 110 \%$, and $115 \%$. If $\alpha$ is $100 \%$ then the price is not changed. $\beta$, the strategic parameter for defrost, is selected from the following 7 values: $92.5 \%$, 95\%, 97.5\%, 100\%, 102.5\%, $105 \%$, and $107.5 \%$. If $\beta$ is $100 \%$ then the price is not changed. One of the stock strategies mentioned previously is selected. $\gamma$, the strategic parameter for stock, is selected from the following 3 values: 1.1, 1.2, and 1,3

We make a strategy number from these three kinds of strategic parameters. This set called as strategy number, and the strategy number $\operatorname{Tn}(i)$ of the agent $i$ will be expressed as following.

$\operatorname{Tn}(i)=1 \times \operatorname{Str} 1(i)+7 \times \operatorname{Str} 2(i)+49 \times \operatorname{Str} 3(i)$

Here, $\operatorname{Str} 1(i), \operatorname{Str} 2(i)$, and $\operatorname{Str} 3(i)$ are integers. Str1(i) represents the defrost strategy, from 0 to 6 . In $\operatorname{Str} 1(i), 0$ corresponds to $92.5 \%$ and 6 is $107.5 \%$ of $\beta$. Str $2(i)$ is the price strategy and it also is 0 to 6 . In Str $2(i)$, 0 corresponds to $97.5 \%$ of $\mathbf{a}$, and 6 corresponds to $110 \%$. Str $3(i)$ is the stock strategy, from 0 to 2. In $\operatorname{Str} 3(i), 0$ corresponds to 1.1 and 2 is 1.3. The agent's strategy is expressed by combining the above-mentioned parameters. The agent has a total of 147 different strategies.

\subsection{Strategy Adjustment through Log Data}

The agent's decision-making rules are based on the data from previous rounds. Without strategic parameters, the agent just adapts to the past situation, and it is not enough to be worthy rival of the human players. One of the ways to find good strategy parameters is that the agent uses a model of game world that includes the prediction of the future customers and other players' actions. However, to implement the agent this kind of model to agent is difficult because of the property of the game. Furthermore, the size of the demands and the total number of players are usually changed case by case. We focus on the property of the Bakery Game. As we mentioned, there is the optimal number of customers calculated by model B. This optimal number of customers can be calculated without effect of the number of players, and it varies according to the state of the market.
The agent tests its strategic parameter on the log data. A log data is a recorded players' decision, and we can regenerate game by using the log data to the game system.

Using optimal number of the customer and log data, the set of the strategic parameters depending on the situation are decided. At first, the agent selects a round. The agent recalculates the surplus of all of the 147 strategies from log data until the selected rounds. The surplus of the previous three rounds in the past including the chosen round are the record as the score of the strategy. After scoring all strategy of the selected round, the agent selects the best strategy. The agent records the strategy with the optimal number of the customer in the previous three rounds. The set of the strategy and the optimal number of the customers are kept to the experience data pool. The agent selects the all of the rounds in the log data and gathers the result to data pool.

Agent uses the set of the strategy with the optimal number of the customer as follows. The agent compares the difference between the optimal numbers of customers of the last two rounds with that in the experience data pool. The comparison of the current optimal number of customers and the recorded optimal number of the customer are written as following.

$$
D s t_{k}=\left|C o_{t-1}-R C o_{2}{ }_{k}\right|+\left|C o_{t-2}-R C o_{1_{k}}\right|
$$

Here, $D s t_{k}$ is the difference of the current data and recorded data $k . \mathrm{Co}_{t}$ is the stock amount in time $t$. $R \mathrm{CO}_{1_{k}}$ means the recorded optimal number of customer in the last previous round. $\mathrm{RCO}_{2} \mathrm{k}_{\mathrm{k}}$ means the recorded optimal number of customers in the round before last round.

The agent can choose the strategy that considers next round's surplus in use of this method. The outline of the using of strategy is shown in Fig. 2.

\section{EXPERIMENT}

\subsection{Experimental Set Up}

This paper uses three kinds of log data in order to test agent in various gaming situation. These log data are called as Case A, Case B, and Case C. Each log data include five rounds of decision-making parameters and the change of total number of customers. There are 12 
players in Case A and Case C. There are 10 players in Case B.

Before gaming simulation, agents calculate the change of the optimal number of customers and surplus all of the strategy. The agent's set of optimal number of customers and strategy are shown in Table 1.

The strategic parameter of price $\alpha$, the strategic parameter of defrost $\beta$, and the strategic parameter of $\gamma$ are tested and evaluated by the surplus in the three kinds of log data. The agent joins the remaining log data with the winning strategy.

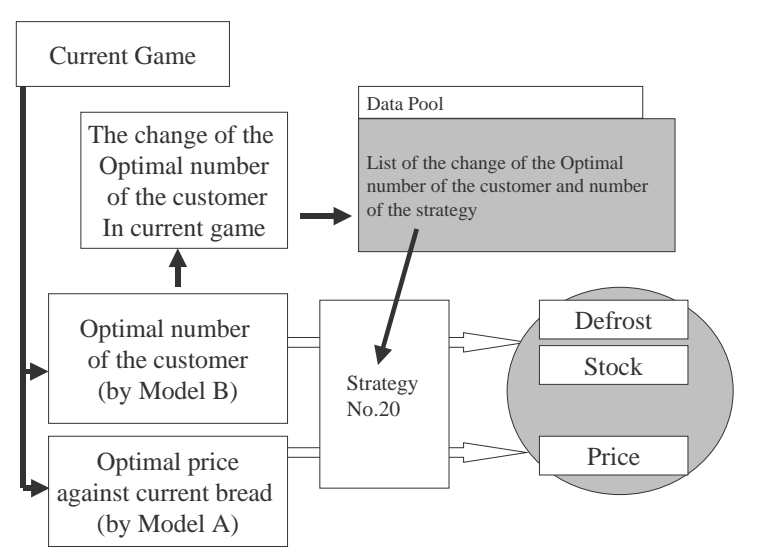

Fig. 2. Outline of the use of strategy

Table 1. Found set of the optimal number of the

\begin{tabular}{|c|c|c|c|c|}
\hline & $\begin{array}{l}\text { Optimal } \\
\text { number of } \\
\text { customer } \\
\text { for t- } 2\end{array}$ & $\begin{array}{l}\text { Optimal } \\
\text { number of } \\
\text { customer } \\
\text { for } t-1\end{array}$ & $\begin{array}{l}\text { Optimal } \\
\text { number of } \\
\text { customer } \\
\text { in the } \\
\text { experience } \\
\text { (not used) }\end{array}$ & $\begin{array}{c}\text { Strategy } \\
\text { number }\end{array}$ \\
\hline \multirow{3}{*}{ Case A } & 100 & 127 & 128 & 63 \\
\hline & 127 & 128 & 125 & 67 \\
\hline & 128 & 100 & 124 & 0 \\
\hline \multirow{3}{*}{ Case B } & 100 & 112 & 120 & 1 \\
\hline & 112 & 120 & 102 & 7 \\
\hline & 120 & 102 & 117 & 0 \\
\hline \multirow{3}{*}{ Case C } & 100 & 100 & 128 & 14 \\
\hline & 100 & 128 & 123 & 14 \\
\hline & 128 & 123 & 145 & 14 \\
\hline
\end{tabular}

For agent, the game on log data is the same as gaming simulation against human players. The agent calculates the optimal number in the previous rounds and compare to the change of the optimal number of customers to find suitable strategy in the data pool.

We initialize the agent following the game scenario. All of the market customers' history is set to 1200 in case there are 12 players. If there are 10 players, the history of the customers is set to 1000. In all cases, agent occupies the last player on the log. Initial customer changing ratio is set to 1.1 .

\subsection{Results}

Figure 3 shows the result of the gaming on Case A with the experience of Case B, and Case C. In this figure, agent becomes the winner of the game. Figure 4 shows the result of the gaming by using the experience of Case $A$, and Case C. In this figure, the agent could not become the winner of the game, but its surplus increases is similar to the winner player. Figure 5 shows the result of the gaming by using the experience of Case A, and Case B. In this figure, the agent also becomes the winner of the game at fifth round.

\subsection{Discussion}

From Fig. 3 and Fig. 5, the agent can be the winner of the game. In the Fig. 4, the agent becomes the third in the round 5 . In this case, the agent did not increase its surplus in the round 3. Except the round 3, its behavior is similar to that of winner.

From viewpoint of the facilitator of Business Game, this agent is useful because of two reasons. First, the score of the agent keeps the top group of the players. The proposed agent model has possibility to become worthy rival of human players. The second is that the agent can make decision quickly. The agent prepares the strategy set before gaming, and once the game begins, the agent works in real-time. This is important because during the game, the facilitator must take care of the players not only the agents.

The proposed agent has another merit. This agent is designed by combining two ideas. One is the rule of the simple decision-making that depends on the character of the game. The other is the strategy from the experience. Considering many kinds of gaming simulation we observe, it is difficult to construct the agent model for each game. In this paper, we have shown that using experience a strong agent can be designed more easily by the using proposed structure.

\section{CONCLUSIONS AND REMARKS}

In this paper, we proposed an agent model that joins in Bakery Game. The agent has simple decision-making rules and has function to gather strategies from experience. The agent is tested against the log data, and it shows the possibility to be worthy rival of human player. This kind of real-time computer agent is useful for gaming facilitators. 


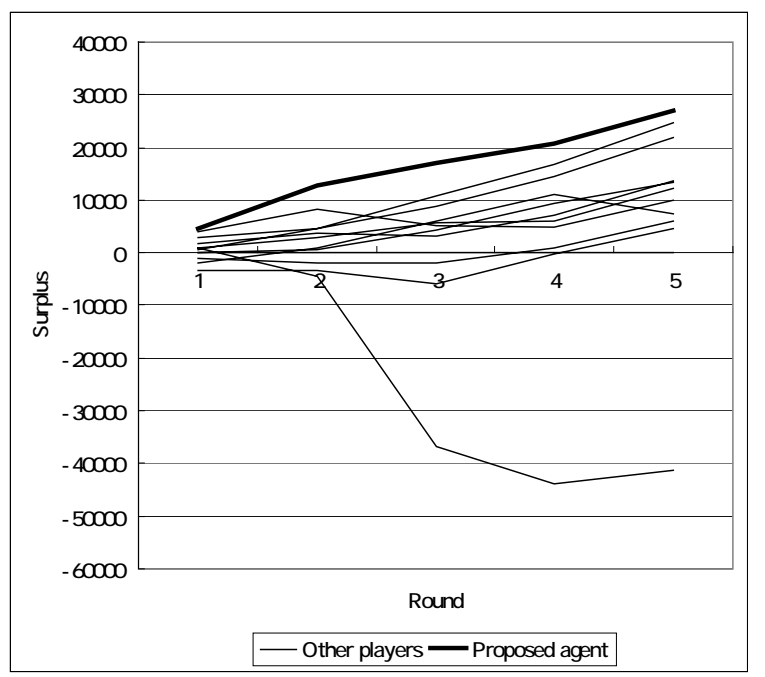

Fig. 3. The change of the surplus on Case A

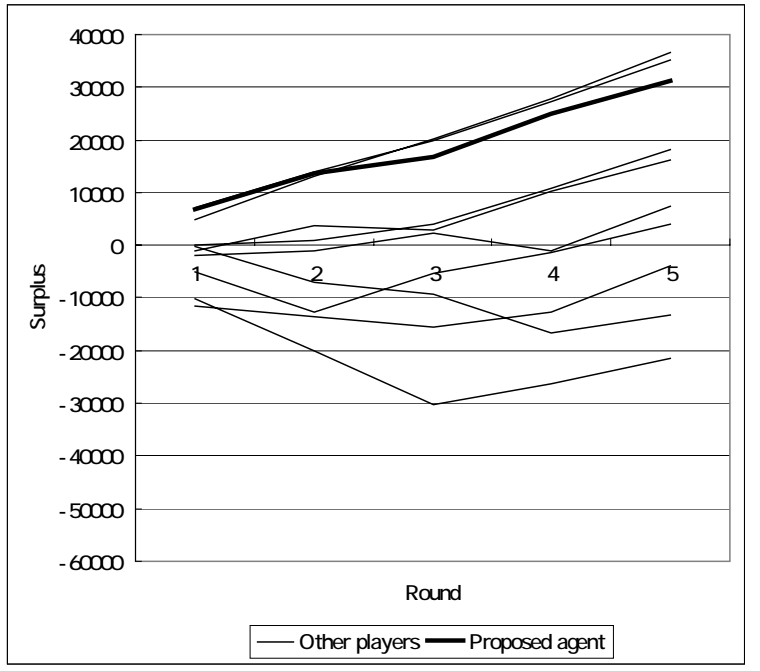

Fig. 4. The change of the surplus on Case B

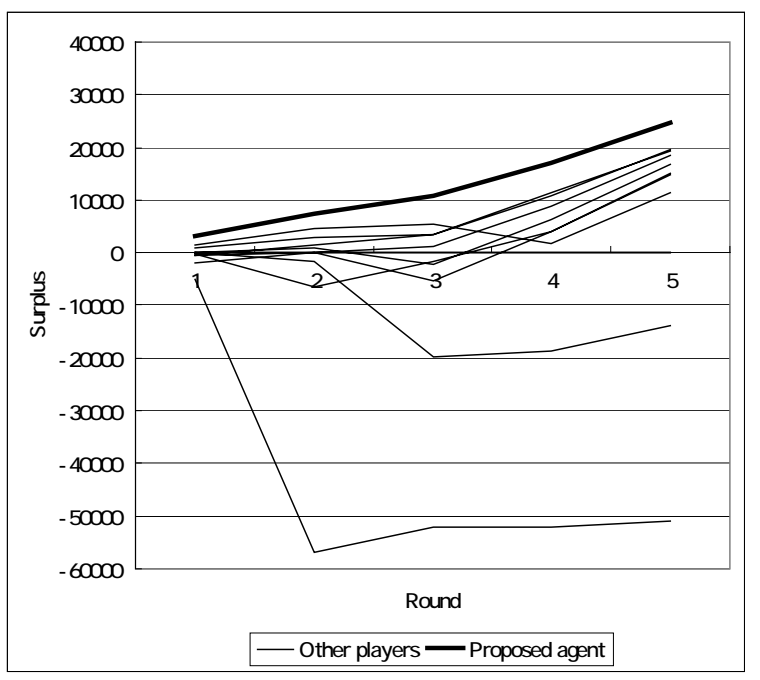

Fig. 5. The change of the surplus on Case $\mathrm{C}$
On the other hand, there is possibility that the human player may change his decision for countermeasure of the agent. In addition, we have only a few log data and the test of the agent is not enough. We suppose that the proposed strategy selection algorithm may not be suitable for a huge number of sets of optimal number of the players and strategy. In the future work, we must join the agent player to actual Gaming Simulation, and modify algorithms.

\section{REFERENCES}

[1] I. Stahl, "What is Operational Gaming ?," Operational Gaming: An International Approach IIASA 1983, pp 25-39, 1983

[2] T.Terano, H.Suzuki, Y.Kuno, H.Fujimori, H.Shirai, C.Nishio, N.Ogura, M.Takahashi, "UNDERSTANDING YOUR BUSINESS THROUGH HOME-MADE SIMULATOR DEVELOPMENT," Paper presented in Proc. ABSEL 1999, pp.65-71, 1999

[3] Kiyoshi Arai, Hiroshi Deguchi, Toshiyuki Kaneda, Fumitoshi Katou, Mieko Nakamura, "Gaming simulation,” Nikkagiren, 1998 (in Japanese)

[4] Hiroaki Shirai "Business model creation method, " Nikkagiren, 2001 (in Japanese)

[5] H.Fujimori, Y.Kuno, H.Shirai, H.Suzuki, T.Terano, ” ALEXANDER ISLANDS: GSSM TINY BUSINESS SIMULATOR ON THE WWW," Paper presented in Proc. ABSEL 1999, pp.224-225, 1999

[6] Joshua M. Epstein, Robert Axtell, "Growing artificial societies: social science from the bottom up," Brookings Institution Press, 1996

[7] Masato Kobayashi Takao Terano, "Exploring Business Strategies by Learning Agents,” Proc. ISAGA 2003, pp.557-566, 2003

[8] N. Baba, T. Kita, Y. Takakawahara, K. Oda, "Computer Gaming System with Neural-network and Genetic Algorithms," Instrument and Control Engineering, Vol.36, 6, pp.434-448, 1997

[9] Yuji Shinoda Mina Ryoke Takao Terano Yoshiteru Nakamori, "Strategy Searching for Business Gaming -Software Agents Work for a Game Designer," Paper presented in Proc. THE INTERNATIONAL SYMPOSIUM ON KNOWLEDGE AND SYSTEMS SCIENCES (KSS'2004), pp.166-171, 2004

[10] B. B. Tregoe, J. W. Zimmerman, "Top Management Strategy -What it is \& How to Make it Work," Simon \& Sheuster, 1980

[11] K. Furukawa, M. Misawa, A. Okumura, Y. Konomi, "Strategic Company," Japan Management Association, 1986 (in Japanese) 